\title{
The Notochord, Notochordal cell and CTGF/CCN-2: ongoing activity from development through maturation
}

\author{
W. Mark Erwin
}

Received: 15 August 2008 / Accepted: 30 October 2008 / Published online: 12 November 2008

(C) The Author(s) 2008. This article is published with open access at Springerlink.com

\begin{abstract}
The growth regulating factor $\mathrm{CTGF} / \mathrm{CCN}-2$ is an integral factor in growth and development, connective tissue maintenance, wound repair and cell cycle regulation. It has recently been reported that $\mathrm{CTGF} / \mathrm{CCN}-2$ is involved in very early development having been detected in early notochord formation in zebrafish using $\mathrm{CTGF} / \mathrm{CCN}-2$ promoter-driven green fluorescent protein (GFP) plasmids. In these studies fluorescence was detected early in the developing embryos, a finding of considerable significance in that CTGF/CCN-2 deficient mutant mice die early after birth due to severe cartilage and skeletal dysplasia and respiratory failure. Such findings confirm the importance of $\mathrm{CTGF} / \mathrm{CCN}-2$ in development and of the necessary and sufficient role of this molecule in formation of the skeleton, extracellular matrix and chondrogenesis. Of particular relevance to the relationship between the notochordal cell and CTGF/CCN-2 there is a remarkable sub-species of canine, the 'non-chondrodystrophic' canine that is protected from developing degenerative disc disease (DDD). These animals are unique in that they preserve the population of notochordal cells within their disc nucleus (NP) and these cells secrete $\mathrm{CTGF} / \mathrm{CCN}-2$. We have detected CTGF/CCN2 within conditioned medium developed from the notochordal cells of these animals (NCCM) and used this conditioned medium to demonstrate robustly increased proteoglycan production. The addition of recombinant human $\mathrm{CTGF} / \mathrm{CCN}-2$ to totally serum-free media containing cultures of bovine NP cells replicated the robustly increased aggrecan gene expression found with NCCM
\end{abstract}

W. M. Erwin $(\bowtie)$

Division of Orthopaedic Surgery,

University of Toronto, Toronto Western Hospital,

399 Bathurst St., McLaughlin Pavilion Rm 13-415,

Toronto, ON M5T 2S8, USA

e-mail: mark.erwin@utoronto.ca alone strongly suggesting the importance of the effect of $\mathrm{CTGF} / \mathrm{CCN}-2$ in notochordal cell biology within the disc nucleus of non-chondrodystrophic canines. The chondrodystrophic canine, another sub-species on the other hand are almost totally devoid of notochordal cells and they develop DDD profoundly and early. These two sub-species of canine reflect a naturally occurring animal model that is an excellent example of differential notochordal cell survival and possible associated developmental differences in extracellular maintenance.

Keywords CTGF/CCN-2 $\cdot$ Notochordal cells . Intervertebral disc $\cdot$ Development $\cdot$ Extracellular matrix

\section{Introduction}

The early 1990's marked the discovery and publication of numerous accounts concerning a host of protein products of genes in the $35-40 \mathrm{kDa}$ range consisting of $\mathrm{CTGF} / \mathrm{CCN}-2$ (connective tissue growth factor), Nov, WISP-2 and WISP3 , cyr-6. These and now some others have now been classified as the 'CCN family' of genes after the early suggestion that members of this family of genes acted as 'growth regulators' (Peer 1993; Moussad and Brigstock 2000; Brigstock 2003). CTGF/CCN-2 in particular has in the past been referred to as a growth factor as its earlier name suggests, however the collective work of a number of laboratories and in particular the development of knockout mice has confirmed that $\mathrm{CTGF} / \mathrm{CCN}-2$ for example more accurately serves as a 'modifier' of the signals transmitted by other molecules-such as TGF- $\beta$ (Moussad and Brigstock 2000; Brigstock 2003; Leask and Abraham 2006; Ivkovic et al. 2003). It has also emerged and is fairly broadly accepted within the CCN community that the best designa- 
tion for this molecule is ' $\mathrm{CCN} 2$ '-therefore this designation will remain consistent throughout this review. It is now widely accepted that $\mathrm{CCN}-2$ exerts important homeostatic control of connective tissues by virtue of its ability to mediate extracellular matrix processes. $\mathrm{CCN}-2$ proteins modulate mitosis, cellular migration, binding of other growth factors, wound healing and have varying influence concerning either pro- or anti-apoptotic signaling depending upon the cells and tissues involved (Leask and Abraham 2006; Gygi et al. 2003; Croci et al. 2004; Hishikawa et al. 2000). Over-expression of CCN-2 leads to significant morbidity such as is the case with fibrotic diseases such as may be the case with scleroderma and fibrosis affecting other organ systems such as the liver, kidney, lung, pancreas and others (Moussad and Brigstock 2000; Ivkovic et al. 2003; Perbal 2004; Ming-Jyun et al. 2006). Furthermore, elevated CCN2 expression has been reported to play a crucial role in migratory/invasive processes in breast cancer (Pai-Sheng et al. 2007). On the other hand, human lung adenocarcinoma cells stably transfected with vectors containing CCN2 and injected into nude mice were found to resist metastatic activity. In this study it was theorized that $\mathrm{CCN}-2$ induced inhibition of metastasis may have been the result of inhibiting VEGF-A - dependent angiogenesis-perhaps as a consequence of increasing the degradation of HIF-1 (Cheng-Chi et al. 2006). Therefore it has emerged that $\mathrm{CCN}-2$ has widely diverse functions and is associated with a broad range of physiological activities. One area that has received far less attention has been the dynamic role of CCN-2 in development, particularly with respect to the varying influence of this molecule from early embryogenesis through to the mature organism in various tissue compartments and its contribution to health and disease as a function of its changing expression patterns.

\section{The role of the Notochord in development}

The notochord is common to all higher vertebrates as a developmentally vital structure. During development the notochord serves to define the longitudinal axis and provide 'stiffness' to the developing embryo-as well as to function as a source of differentiation signals to local undifferentiated mesenchymal tissues (Ming-Jyun et al. 2006; Smits and Lefebvre 2003; Marcelle et al. 1999; Barrionuevo et al. 2006; Hirano and Najita 1995). The notochord is of mesodermal origin and forms during the gastrulation phase of the developing embryo where the early notochord begins as at the primitive pit and ultimately becomes a rod-like structure that is enveloped within a sheath composed of predominantly extracellular matrix, collagens and proteoglycans, all of which are similar to the composition of cartilage and mesenchymal/fibrous tissues (Smits and
Lefebvre 2003; Barrionuevo et al. 2006; Cappello et al. 2006). The notochord most closely resembles cartilage in terms of its tissue type and as such the stiffness it provides during development provides for an axial skeleton-like structure until somitogenesis provides for the development of the axial skeleton (Stemple 2005). Signals secreted by the notochord are required in order for sclerotomal cells to migrate, condense and differentiate-all of which are central to the formation of the vertebral column and the developing spinal cord (Hirano and Najita 1995).

The spinal cord is formed from condensation of neural crest cells that migrate and lie dorsal to the notochord, forming the neural tube and ultimately the spinal cord (Echelard et al. 1993; Hirano et al 1996). The terminal stage of notochord development in higher animals is marked by the notochord segmenting during vertebrogenesis and in the vertebral regions it is largely replaced by bone and in the intervertebral areas it forms the centre of the intervertebral disc-the nucleus pulposus (Stemple 2004, 2005).

A number of factors are involved in the complex process of notochordal evolution specifically including the expression of the brachury gene $T$ that codes for the T-box transcription factor-commonly referred to as " $\mathrm{T}$ ", as well as Sonic Hedgehog (SHH), Noggin and Paxl all of which play essential roles that induce differentiation of the sclerotome (Barrionuevo et al 2006; Ghosh 1988a). The $T$ gene is expressed in all mesodermal cells and is expressed within the notochord until formation of the developing embryonic body is completed (Herrmann and Kispert 1994). T however is expressed only transiently by other mesodermal cells demonstrating the directed activity of this gene in notochordal development (Herrmann and Kispert 1994). In mutant mouse studies it has been found that $T$ is necessary for the maintenance of the notochord and that $T$ seems to be a controlling factor in cell motility and/or cell adhesion (Herrmann and Kispert 1994). Interestingly $\mathrm{T}$ protein is able to switch the fate of cells from an ectodermal to a ventral mesodermal destination allowing ventral mesodermal cells to respond to diffusible dorsal signal factors. As the following sections detail, there is an early role for CCN2 in development and in the formation of the notochord in particular. It is therefore an attractive notion that $\mathrm{CCN}-2$ may well interact with such important differentiation and signaling pathways such as those involving $\mathrm{T}$ protein as well as other critical transcription factors such as those encoded by the Sox genes. For example, it has been shown that $\mathrm{CCN}-2$ interacts with WNT signaling in Xenopus development and that it is able to inhibit canonical signaling involving $\beta$-catenin as well as non-canonical signaling probably via interaction with the WNT receptor complex (Mercurio et al. 2004). Further evidence of the complex interaction of $\mathrm{CCN}-2$ with development is that 
overexpression of CCN-2 in Xenopus results in inhibition of neural crest migration-perhaps by virtue of WNT pathway inhibition (Mercurio et al. 2004). The reader is referred to the detailed report by Mercurio et al for further details concerning CCN2 activity and WNT signaling (Mercurio et al. 2004).

Once the sclerotome is formed, in addition to $\mathrm{T}$, the notochord is known to be heavily influenced by several key transcription factors encoded by Sox5, Sox6 and Sox 9 genes (Smits and Lefebvre 2003; Barrionuevo et al. 2006). The Sox expression pattern found in the notochord has similarities to that observed in cartilage where Sox 5, 6 and 9 are coordinated and linked (Lefebvre et al. 2001). The Sox genes encode a number of DNA-binding proteins that are considered to be important relatively late in development as opposed to the earlier stages where they are considered to have some redundancy (Smits and Lefebvre 2003). In general, the Sox DNA binding proteins are thought to perform their various functions in a sterically-defined manner whereby they act as architectural proteins and organize chromatin structure and assemble other DNAbound transcription factors into larger more complex multiprotein units (Wegner 1999). It is likely that Sox proteins link proteins to signal transduction pathways (such as these multi-protein units) which by themselves may not be capable of inducing signal transduction. Sox 5 and 6 gene transcripts are known to be expressed in cartilage but no other tissues in the developing mouse and have some kind of co-operative function with Sox9-considered to be the 'master chondrogenic factor' (Smits and Lefebvre 2003; Lefebvre et al. 2001). Sox 5 and 6 are considered to be crucial to the survival of notochordal cells within the disc nucleus pulposus, a potentially vital pathway inherent within the cells of animals that retain their notochordal cells into adulthood (Moussad and Brigstock 2000; Smits and Lefebvre 2003). Indeed Sox $5^{-/-}$and $6^{--}$mutant mice demonstrate aberrant removal of notochordal cells from the intervertebral areas and do so later than wild type embryos in mice-the theory being that this delay may be associated with a delay in cartilage development and/or apoptosis of notochordal cells (Smits and Lefebvre 2003). These Sox $5^{-1-}$ and $\sigma^{-1}$ mutant mice display a failure to form a nucleus pulposus, along with severely distorted vertebral column development confirming the pivotal role that these genes play in directing the development, maturation and survival of the disc nucleus pulposus (Smits and Lefebvre 2003).

In what is likely a more upstream effect, mutant mice lacking $\operatorname{Sox} 9$ expression fail to develop a normal notochord however the survival of notochordal tissue in the presence of normal Sox9 signaling poses a strong argument for the importance of this transcription factor in notochordal cell maintenance in addition to its known role in chondrogenesis and likely the cooperative interaction of Sox 5, 6, and 9 (Moussad and Brigstock 2000). In addition, Jun (a major transcription factor essential for embryonic development) elicits anti-apoptotic signaling critical to the survival of notochord cells (Croci et al. 2004; Marcelle et al. 1999). However, the precise mechanisms at play in the pivotal morphological evolution of notochordal cells whereby they become located within the nucleus pulposus of the intervertebral disc (IVD) are largely unknown-as is the role played by these cells within the disc (Moussad and Brigstock 2000; Pai-Sheng et al (2007). Clearly there is a delicate and precise 'ballet' involved with the development of this anatomically central structure whereby aberrant expression of any of these elements create a highly disturbed phenotype.

\section{CCN-2 and the Notochord}

With respect to the role played by $\mathrm{CCN}-2$ during development, a recent study introduced anti-sense oligonucleotides into zebrafish embryos to in order to specifically knockdown CCN-2 expression (Ming-Jyun et al 2006). Further these investigators also injected a series of $\mathrm{CCN}-2$ promoter-driven green fluorescent protein (GFP) plasmids into one-cell-stage zebrafish embryos in order monitor the expression of CCN-2. The targeted knockdown of CCN-2 led to a malformed and distorted notochord in the developing embryo and many of the embryos died in early developmental time periods (Ming-Jyun et al 2006). The promoter-driven GFP signal was detected in the midline, floor plate and head section of the notochord of the developing embryos confirming the expression of CCN-2 during embryological development in general and the notochord specifically. The detection of CCN2 linked GFP within the midline, floor plate and head section of these developing embryos is all the more intriguing when taken in context with the findings that $\mathrm{T}$ is considered to be involved with cellular functions concerning cell adhesion (Herrmann and Kispert 1994). CCN-2 is well known to be highly involved in cell growth, migration and adhesion and although there are no direct references in this regard, the detection of GFP-linkedCCN-2 in the developing notochord provides for the notion that an axis involving $\mathrm{CCN}-2$ and $\mathrm{T}$ in notochord development and maintenance may be more complex than is currently understood.

These findings are of considerable relevance in the context of the lethal phenotype generated by the deletion of CCN-2 in mice that results in severe skeletal dimorphisms that is also inconsistent with life shortly after birth (Ivkovic et al. 2003). These CCN-2 deletion mutants are born with severe skeletal abnormalities, malformed rib cages and die due to poor extracellular matrix synthesis, defective cartilage and associated respiratory failure (Ivkovic et al. 2003; Ming-Jyun et al 2006). However, there is as of yet no 
detailed description of the fate of the intervertebral disc nucleus pulposus-in particular what transpires in terms of notochordal cell development apart from impaired skeletogenesis.

With respect to initial notochordal signaling, it is widely recognized in studies involving zebrafish, drosophila and chicks that $S H H$ is a key inductive signal for ventral patterning of the CNS and that a gradient of signaling seems to exist between dorsal ectodermal and ventral mesodermal signaling in CNS development (Horne 2004). The floor plate releases $S H H$ which results in neuronal patterning that are directly related to the gradient of $\mathrm{SHH}$ signals and their interaction with undifferentiated mesenchymal cells. It was as a consequence of these important experiments that $S H H$ became known as a classic morphogen. It has been reported in other studies that the CCN-2 regulatory region has a shared homology within the zebra fish $S H H$ gene; further lending support to the essential involvement of $\mathrm{CCN} 2$ in key developmental processes involving embryological developmental patterning (MingJyun et al. 2006).

Notochordal cells and the intervertebral disc

It has been reported that coincident with the reduction in the number of notochord cells, the nucleus pulposus is seen to undergo typical internal disorganization accompanied by degenerative changes (Ghosh 1988b; Thompson et al. 1991; Aguiar et al. 1999). However, animals that retain notochordal cells within their IVD NP do not develop degenerative disc disease nearly as early or as severely as those animals that do not retain these cells. There are natural occurring sub-species of canine that are unique in that one, the non-chondrodystrophic (NCD) canine is protected from developing degenerative disc disease (DDD), whereas the chondrodystrophic (CD) canine (beagles, dachshunds, poodles) suffer significant and early DDD (Braund 1975). The single most critical difference between these two sub-species is that the non-chondrodystrophic canine maintains its population of notochordal cells within the nucleus pulposus (NP) of the IVD for most of the life of the animal whereas the CD canine contains sparse notochordal cells by even 1 year of age. During the course of harvesting notochordal cells from canine sources the number of notochordal cells available is strikingly different between these sub-species. The experience of this writer is such that 30 lumbar IVD NP's harvested from five (5) 8-12 month old NCD canines (6 lumbar discs each) will typically yield $40-45 \times 10^{6}$ notochordal cells. Eight (8) CD canines on the other hand using 6 lumbar IVD NPs obtained from each will typically yield only $3-4 \times 10^{6}$ notochordal cells. The disorganization and degenerative changes witnessed in notochordal cell-poor animals strong- ly suggests that notochord cells when present, act as matrix 'guardians' that continue to secrete growth and other factors therefore modulating a homeostatic protective action on the disc matrix (Aguiar et al. 1999; Oegema 2002).

Aguiar, Johnston and Oegema were the first amongst a very few to suggest that notochordal cells were able to biochemically contribute to IVD homeostasis by developing a crude conditioned medium and evaluating proteoglycan production in bovine disc-derived NP cells (Aguiar et al. 1999). This first study by this group was followed by our own studies of this relationship. In one study we reported that totally serum-free notochordal cell conditioned medium (NCCM) obtained from NCD notochordal cells could in a dose-dependent fashion, upregulate proteoglycan production and in a non-dose-dependent fashion increase cell proliferation in bovine caudal disc NP cells (Erwin and Inman 2006). In a second study, also using bovine caudal disc NP as the target cells, we demonstrated upregulated gene expression for the essential extracellular matrix proteoglycans aggrecan and versican after 24-hour culture with totally serum-free notochordal cell conditioned medium (Erwin et al. 2006). In addition, gene expression

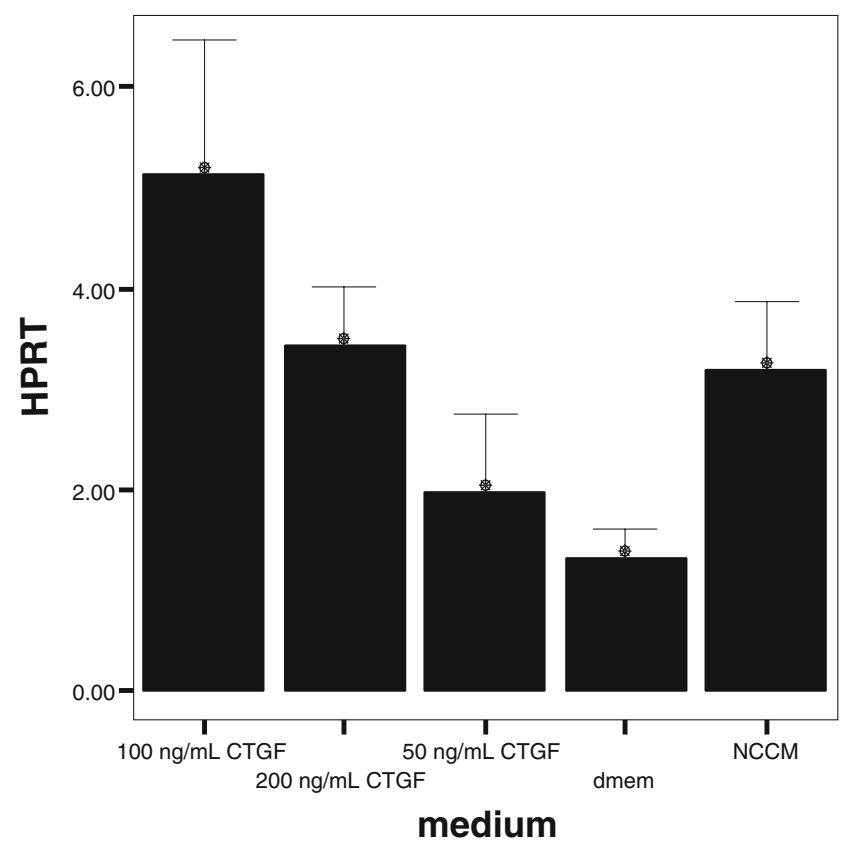

Fig. 1 Aggrecan gene expression (normalized to HPRT) for discderived chondrocytes. Chondrocytes were cultured for $24 \mathrm{~h}$ with either, DMEM, $50 \mathrm{ng} / \mathrm{ml}, 100 \mathrm{ng} / \mathrm{ml}, 200 \mathrm{ng} / \mathrm{ml} \mathrm{rCTGF}$ and NCCM. Amplicons were generated from the reverse transcription and subsequent PCR using aggrecan specific primers of $1 \mu \mathrm{g}$ total RNA harvested from treated chondrocytes (Trizol). The above results reflect mean gene expression ratios of between 5 and 9 separate experiments all from at least three separate sources of chondrocytes and notochord cells. DMEM and CTGF 50 were repeated three times, CTGF 100 and 200 were repeated eight times, and NCCM was repeated nine times. (This figure from Erwin et al. 2006) 


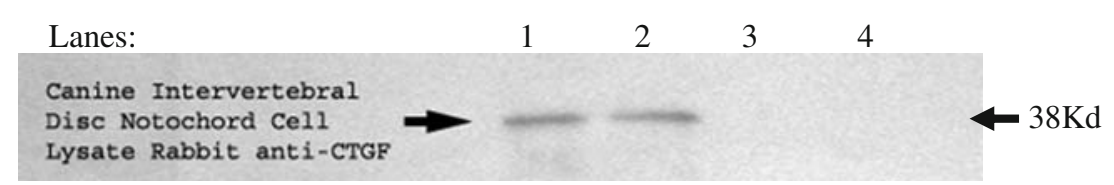

Fig. $210 \mu \mathrm{g}$ Notochordal cell lysates run in parallel using rabbit anti-CTGF Western Blot (black arrowhead) lanes 1-2. Control lanes were murine $\beta$-cells, lanes $3-4$

for hyaluronic acid synthase-2, the enzyme essential for hyaluronic acid synthesis production was also upregulated in bovine disc NP cells. Using LC-MS/MS mass spectroscopy we identified the presence of $\mathrm{CCN}-2$ within the conditioned medium (Erwin et al. 2006). After we detected CCN-2 within the notochordal cell conditioned medium, we cultured bovine NP cells with serum-free notochordal cell conditioned medium (NCCM) and measured doses of recombinant human CCN2 (rCCN2) (a kind gift from Dr. David Brigstock, Ohio State University) and compared aggrecan gene expression by the bovine NP cells. We found that $100-200 \mathrm{ng} / \mathrm{mL}$ of $\mathrm{rCCN} 2$ induced upregulation of aggrecan gene expression to exactly the same level of expression as our serum free NCCM derived from NCD canine-source notochordal cells (Fig. 1) Erwin et al. 2006). We have recently followed-up our mass spectroscopy findings and have verified the presence of CCN2 in NCD notochordal cell lysates using Western blotting techniques (antibody a kind gift from Dr. Andrew Leask, University of

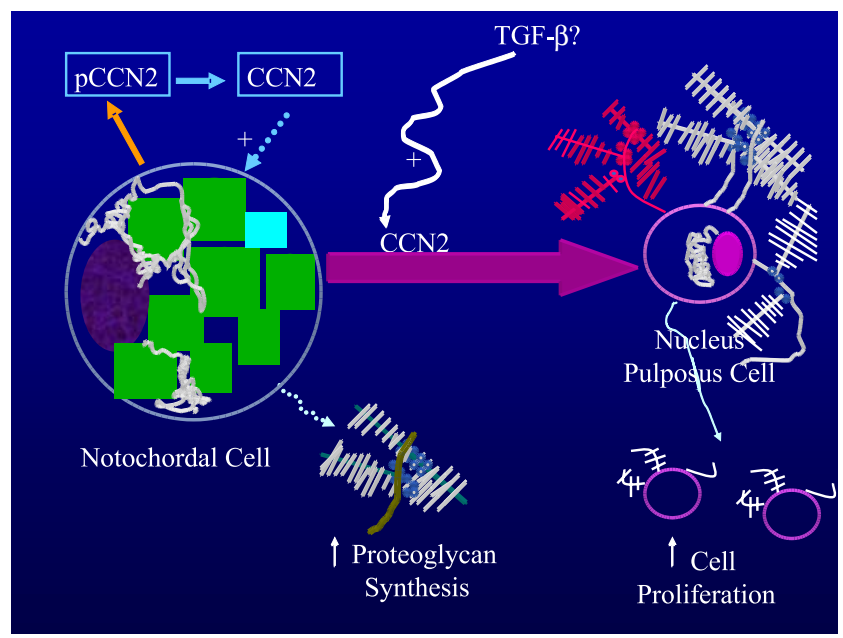

Fig. 3 Schematic depicting notochordal cell interaction with nucleus pulposus cells. The notochordal cell secretes a pro-form of CCN2 that is likely activated within the cytoplasm to the active form. CCN2 acts in a paracrine fashion upon nucleus pulposus cells. The possible role of TGF- $\beta$ within this notochordal cell/nucleus pulposus cell milieu is a question at the present. CCN2 is known to be a downstream effector molecule of TGB- $\beta$ signaling-however the specific role of TGF- $\beta$ within this pathway has not yet been tested. Notochordal cells secrete a unique form of aggrecan themselves (depicted as notochordal cell secreted increased proteoglycan synthesis). Also, soluble factors secreted by notochordal cells activate nucleus pulposus cells to increase their proteoglycan production (indicated as increased proteoglycan synthesis by nucleus pulposus cells in red)
Western Ontario) (Fig. 2). It appears from our initial studies that notochordal cells secrete the pro-form of CCN2 that is activated likely within the cytoplasm to in turn act in a paracrine fashion upon nucleus pulposus cells. The question remains concerning the possible autocrine activity of CCN2 since it is known to fulfill both functions-and may well do so within the notochordal cell-rich milieu of the NCD canine nucleus pulposus (Fig. 3).

It has been reported that the NP cells of non-chondrodystrophic canines (notochordal cell-rich) produce proteoglycans that migrate considerably further from the cell prior to becoming immobilized within the extracellular matrix than do proteoglycans produced from notochordal cells of chondrodystrophic dogs (12). In the case of the chondrodystrophic canine, this process may be impeded and therefore hinder the production of a protective peri-cellular matrix and lead to a loss of these otherwise anabolically important cells and increase the rate of degenerative change within the matrix. These differences in proteoglycan biology may explain both structurally and functionally that the notochordal cell-rich disc may afford superior characteristics than the notochordal cell-poor chondrodystrophic disc (Stemple 2005).

\section{Role of apoptosis in DDD}

Apoptosis or programmed cell death is essential to tissue re-modeling and the normal metabolic process of growth, development and tissue homeostasis. CCN2has been described as having both pro-apoptotic and anti-apoptotic properties, depending upon the type of cells and culture environment studied (Lefebvre et al. 2001; Wegner 1999; Horne 2004). It has been reported that inhibiting CCN2 production induces apoptosis of rhabdomyosarcoma cells by removing autocrine $\mathrm{CCN} 2$ signaling whereas the addition of CTGF significantly increased cell survival (Wegner 1999). On the other hand, it has been reported that CCN2 induces apoptosis via the type I caspase-3 pathway in human aortic smooth muscle cells (Ghosh 1988b). The notochordal cell-rich IVD NP resists degenerative change whereas notochordal cell-poor discs do not. Perhaps part of the resistance to degenerative change on the part of notochordal cell-rich discs is afforded by prosurvival signals secreted by these cells. Since CTGF is known to promote ECM synthesis, cell proliferation, proteoglycan and collagen production, our discovery that 
notochordal cells secrete CCN2 may figure prominently in the resistance of certain species to the development of DDD as well as possibly fulfilling an anti-apoptotic function.

The ongoing secretion of $\mathrm{CCN}-2$ on the part of notochordal cells found within the mature mammalian intervertebral disc such as the NCD canine is a fascinating phenomenon. The mechanisms at play within this highly compartmentalized tissue continue to remain largely unexplored. However, unlike fibrotic disease whereby upregulated $\mathrm{CCN}-2$ signaling induces pathological consequences it may be that within the IVD nucleus pulposus CCN-2 signaling is a highly adaptive response that provides a favorable environment for long-term homeostasis. The likely genetic mechanisms responsible for the ongoing survival of notochordal cells within the NCD canine disc as opposed to the CD canine disc are not known. Further, the heterogeneity of nucleus pulposus and notochordal cells continue to poorly characterized-there is no definitive marker for notochordal cells.

The role played by $\mathrm{CCN}-2$ in the development of the notochord has been partially established in mice and lower animals such as zebrafish. This tends to occur at times when the developing embryo is in a state of flux, such as when it lacks a mature circulatory system. However little is known concerning the biology of notochordal cells that naturally persist within the avascular IVD nucleus pulposus within the mature mammal such as the is the case of the NCD and CD canines. It is likely that the multifunctional role played by $\mathrm{CCN}-2$ exerts other effects upon the extracellular matrix milieu and may be a key player in the maintenance of a healthy compartmentalized tissue such as the IVD nucleus. The IVD is an immune privileged, avascular tissue compartment which is a very different environment than is encountered in most other parts of the body. However there continues to be no explanation for the persistence of notochordal cells within this compartment by some animals and not others. Yet such persistence is clearly associated with superior tissue integrity and protection from degradation. It may be that certain essential molecular and signaling switches are activated in the development and maturation of the notochord in some animals such as the NCD canine thus intimately involving CCN-2. Such molecular switching in turn may allow these cells to survive through maturity and in so doing, preserve matrix integrity within the fully differentiated tissue compartment such as the IVD nucleus pulposus. To date however this question remains a perplexing and fascinating area yet to be explored.

\section{Summary}

There are many open questions concerning the role played by CCN-2 both in development and in maturity in a host of physiological processes. Taken together with a number of other reports $\mathrm{CTGF} / \mathrm{CCN}-2$ appears to play a pivotal role within numerous signaling pathways that are important in development, cellular differentiation, cell migration, proliferation, cell adhesion, and a host of other crucial cellular regulatory functions. As highlighted earlier in this paper GFP-conjugated CCN2 expression has been detected very early in development in the zebrafish notochord. The detection of CCN2 activity at the critical time when the notochord is undergoing its earliest organization and differentiation when taken in context with the capacity for CCN2 to suppress WNT signaling suggests the tantalizing notion that CCN2 could occupy an unknown yet pivotal role much earlier in development than current studies have shown. The situation involving CCN2 within the canine adult intervertebral disc nucleus pulposus represents something of an enigma; perhaps in this case mature tissue within an isolated and avascular tissue compartment provides a unique physiological niche whereby under certain circumstances, sustained ongoing secretion of $\mathrm{CCN}-2$ is more friend than foe.

Open Access This article is distributed under the terms of the Creative Commons Attribution Noncommercial License which permits any noncommercial use, distribution, and reproduction in any medium, provided the original author(s) and source are credited.

\section{References}

Aguiar DJ, Johnson SL, Oegema TR Jr (1999) Notochordal cells interact with nucleus pulposus cells: regulation of proteoglycan synthesis. Exp Cell Res 246:129-137 doi:10.1006/ excr.1998.4287

Barrionuevo F (2006) Sox 9 is required for notchord maintenance in mice. Dev Biol 295:128-140 doi:10.1016/j.ydbio.2006.03.014

Braund KG (1975) Morphological studies of the canine intervertebral disc. The assignment of the beagle to the achondroplastic classification. Res Vet Sci 19:167-172

Brigstock DR (2003) The CCN family: a new stimulus package. J Endocrinol 178:169-175 doi:10.1677/joe.0.1780169

Cappello R (2006) Notochordal cell produce and assemble extracellular matrix in a distinct manner, which may be responsible for the maintenance of a healthy nucleus pulposus. Spine 31(8):873882 April 15-31

Chang C-C et al (2006) Effect of connective tissue growth factor on hypoxia-inducible factor 1a degradation and tumor angiogenesis. J Natl Cancer Inst 98(14):984-995

Croci $\mathrm{S}$ et al (2004) Inhibition of connective tissue growth factor (CTGF/CCN2) expression decreasess the survival and myogenic differentiation of human rhabdomyosarcoma cells. Cancer Res 64:1730-1736 doi:10.1158/0008-5472.CAN-3502-02

Echelard Y et al (1993) Sonic hedgehog, a member of a family of putative signaling molecules, is implicated in the regulation of CNS polarity. Cell 75:1417-1430 December 31

Erwin WM, Inman RD (2006) Notochord cells regulate intervertebral disc chondrocyte proteoglycan production and cell proliferation. Spine 31(10):1094-1099 May 
Erwin WM (2006) Nucleus pulposus notochord cells secrete connective tissue growth factor and up-regulate proteoglycan expression by intervertebral disc chondrocytes. Arthritis and Rheumatism 54 (12):3859-3867 Dec

Ghosh P (1988a) The biology of the intervertebral disc 1. CRC, Boca Raton Florida

Ghosh P (1988b) The biology of the intervetebral disc 2, vol. 2. CRC, Boca Raton Florida

Gygi D (2003) Human connective tissue growth factor expressed in Escherichia coli is a non-mitogenic inhibitor of apoptosis. Biochem Biophys Res Commun 311:685-690 doi:10.1016/j. bbrc.2003.10.061

Herrmann BG, Kispert A (1994) The T genes in embryogenesis. Trends Genet 10(8):280-286 doi:10.1016/0168-9525(90)90011-T

Hirano SHR, Najita M (1995) Morphological analysis of the role of the neural tube and notochord in the development of somites. Anat Embryol (Berl) 192:445-457 doi:10.1007/BF00240377

Hirano S (1996) Morphological analysis of the early development of the chick neural tube separated from the floor plate and notochord. Exp Neurol 139(2):317-321 doi:10.1006/ exnr.1996.0105

Hishikawa K, Nakaki T, Tomoko F (2000) Connective Tissue Growth Factor induces apoptosis via caspace- 3 cultured human aortic smooth muscle cells. Eur Spine J 392:19-22

Horne J (2004) Sonic hedgehog, the morphogen. Nat Milestones Dev 1 (July):21-23

Ivkovic S et al (2003) Connective tissue growth factor coordinates chondrogenesis and angiogenesis during skeletal development. Development 130:2779-2791 doi:10.1242/dev.00505

Leask A, Abraham DJ (2006) All in the CCN family: essential matricellular signaling modulators emerge from the bunker. J Cell Sci 119:4803-4810 doi:10.1242/jcs.03270

Lefebvre V, Behringer RR, de Crombrugghe B (2001) L-Sox5, Sox6 and Sox 9 control essential steps of the chondrocyte differentiation pathway. Osteoarthritis Cartilage 9(supplement A):S69-75 doi:10.1053/joca.2001.0447

Marcelle C, Ahlgren S, Bronner-Fraser M (1999) In Vivo regulation of somite differentiation and proliferation by sonic hedgehog. Dev Biol 214:277-287 doi:10.1006/dbio.1999.9389
Mercurio S (2004) Connective tissue growth factor modulates WNT signalling and interacts with the WNT receptor complex. Development 131:2137-2147 doi:10.1242/dev.01045

Ming-Jyun C (2006) The physiological role of CTGF/CCN2 in zebrafish notochord development and biological analysis of the proximal promoter region. Biochem Biophys Res Commun 349:750-758 doi:10.1016/j.bbrc.2006.08.095

Moussad EEA, Brigstock DR (2000) Connective tissue growth factor: What's in a name? Mol Genet Metab 71:276-292 doi:10.1006/ mgme.2000.3059

Oegema TR Jr (2002) The role of disc cell heterogeneity in determining disc biochemistry: a speculation. Biochem Soc Trans 30(6):839-844 doi:10.1042/BST0300839

Pai-Sheng C et al (2007) CTGF enhances the motility of breast cancer cells via an integrin-avB3-ERK1/2 dependent S100A4-upregulated pathway. J Cell Sci 120:2053-2065

Peer B (1993) The modular architecture of a new family of growth regulators related to connective tissue growth factor. FEBS Lett 327:125-130 doi:10.1016/0014-5793(93)80155-N

Perbal B (2004) CCN proteins: multifunctional signalling regulators. Lancet 363:62-64 doi:10.1016/S0140-6736(03) 15172-0

Smits P, Lefebvre V (2003) Sox5 and Sox6 are required for notochord and extracellular matrix sheath formation, notochord cell survival and development of the nucleus pulposus of intervertebral discs. Development 130:1135-1148 doi:10.1242/ dev.00331

Stemple DL (2004) The notochord. Curr Biol 14(20):R873-R874 doi:10.1016/j.cub.2004.09.065

Stemple DL (2005) Structure and function of the notochord-an essential organ for chordate development. Development 132:2503-2512 doi:10.1242/dev.01812

Thompson JP, Oegema TR Jr, Bradford DS (1991) Stimulation of mature canine intervertebral disc by growth factors. Spine 16(3):253-260 doi:10.1097/00007632-19910300000001

Wegner M (1999) From head to toes: the multiple facets of Sox proteins. Nucleic Acids Res 27(6):1409-1420 doi:10.1093/nar/ 27.6.1409 